\title{
Formalizing collaboration in decision-making: a case study in military epidemiological early warning
}

\author{
Liliane Pellegrin ${ }^{1,2}$ \\ ${ }^{1}$ Département de Télémedecine, \\ Institut de Médecine Navale du Service de Santé des Armées \\ (IMNSSA), TOULON \\ ${ }^{2}$ Laboratoire d'Enseignement et de Recherche sur le \\ Traitement de l'Information Médicale (LERTIM, - EA 3283) \\ Faculté de Medecine, Université de la Méditerranée \\ 1.pellegrin@imnssa.net \\ Charlotte Gaudin $^{2,4}$, Nathalie Bonnardel ${ }^{4,5}$ \\ ${ }^{4}$ Centre de Recherche en Psychologie de la \\ Connaissance, du Langage et de l'Émotion (PSYCLE, \\ E.A- 3273) \\ Université de Provence \\ ${ }^{5}$ Institut Universitaire de France \\ Paris \\ charlottemariegaudin@hotmail.com \\ nathalie.Bonnardel@univ-provence.fr \\ Gaetan Texier $^{2,3}$, Jean Baptiste Meynard ${ }^{2,3}$, Hervé Chaudet ${ }^{2,3}$ \\ ${ }^{3}$ Unité de Recherche Epidémiologique - Département d'Epidémiologie et de Santé Publique \\ Institut de Médecine Tropicale du Service de Santé des Armées (IMTSSA) \\ ${ }^{2}$ Laboratoire d'Enseignement et de Recherche sur le Traitement de l'Information Médicale (LERTIM, - EA 3283) \\ Faculté de Medecine, Université de la Méditerranée \\ gaetex1@gmail.com \\ jb.meynard@wanadoo.fr \\ lhcp@acm.org
}

\begin{abstract}
Motivation -This paper introduces the resolution of a medical complex, uncertain and time-constrainted situation: the early warning outbreak in military deployments. Research approach. We have studied a simulated alarm occurring in the Department of Epidemiology in the Institute of Tropical Medicine of the French Forces, managed by physicians belonging to this department. A task analysis method has been developed to identify and describe components of medical activities, performed by medical teams. This method, called EORCA, is based upon the systematic observation of "event", describing performance of actions by agents. Findings/Design. The results indicate that the situation management is based upon collaborative activities. Managing an epidemiological early warning is a collective and complex activity. It requires numerous information transmissions between members of the team to identify diseases and to prepare adequate actions in a context of uncertainty events and short interval of time.
\end{abstract}

Keywords

Complex and time-constrained situation, medical decision, collaborative work, task coordination, epidemiological early warning system

\section{INTRODUCTION}

Medical decision making belongs to complex and time-pressure activities dealing with uncertainty features depending on the evolution of various parameters (environmental, biological...). Patient management in medical situations, such as emergency or ICU critical care, presents similarities with situations occurring in dynamic, complex and criticalsafety environments. Yet-existing models can be applied to healthcare domains, both for analyzing expert individual decision-making and for representing the medical team's activities. Especially, naturalistic decision models have demonstrated their adequacy to formalize individual or collective decision-making and actions in complex, dynamic environments, including medical situations. In this context, the management of information is a key point for the resolution of a medical complex, uncertain and time-constraint situation by physicians.

This paper presents a specific method developed to identify and describe components of medical activities, performed by a medical team. The task analysis method introduced here associates an analysis and a formal description of a

(C) The Authors 2009. Published by the British Computer Society

Proceedings of NDM9, the 9th International Conference on Naturalistic Decision Making

London, UK, June 2009 
situation work based upon the systematic observation of "events" describing performance of actions by agents. This method has helped us to describe collaborative actions between physicians during medical situation management and we present here the first results we have obtained. We applied this method upon decision-making issued from collaborative experts management of an outbreak detected by a surveillance system for early warning in the French Forces (Chaudet $\mathrm{H}$ et als, 2006). The experienced situation is a simulated alarm occurring in the Department of Epidemiology in the Institute of Tropical Medicine of the French Forces (IMTSSA), managed by physicians belonging to this department. In this medical field, the information managed during the resolution has concerned both individual and collective medical data, environmental and spatio-temporal specificities, military organization and medical resources.

\section{THEORICAL BACKGROUND}

\section{Cooperation and collaboration in medical team}

Research in cognitive psychology and ergonomics applied to medical activities have been related first to individual expert decision-making (Gaba, 1992, Patel et al., 2002) and secondly opened to collective team activities in complex and constrained situations. Especially, the focus has been upon complex and time-restricted situations, such as anaesthesiology, emergency, intensive care units (Nyssen and De Keyser, 1998). Healthcare activities belong to professional situations occurring in dynamic, complex and critical-safety environments implying collective aspects of patient managements (Reddy et al., 2002) and their implication in clinical information systems (Beuscart-Zéphir et al., 2005). Over the past decade, numerous studies in NDM fields of interest have described the process by which expert decisions are made in naturalistic settings. The core issue is that expert decision-makers focus their attention on the comprehension of the situation. Decisions are affected by its contextual constraints and its dynamics. Instead, individual and collective resources are dedicated to understanding the situation and, once understood, the course of action is relatively clear (Lipshitz, et al., 2001). This is particularly true in medical domains. Healthcare decisions have critical conditions to manage as, for example: high risk, severe time pressure, uncertainty of the patient's life status evolution dealing with changes in patients' health states and complex patient charts based upon different indicators, each of them with specific constraints of evolution. For describing the complexity of tasks in emergency medical care, Xiao and the Lotas Group (Xiao et als, 1996) took into consideration four main components: multiple and/or concurrent tasks, uncertainty, changing plans and compressed work procedures due to high workload. This situation requires the intervention of experienced specialists. They are rarely managing alone the situation, but most of the time, are integrated in a multidisciplinary team of healthcare providers. So, the activities of healthcare professionals are not only individual expertise but collective and complex activities involving a set actors with different roles and various skills with different levels of expertise to improve medical situation diagnosis (physicians and analysis labs) or to manage the patient care process (physicians, nurses...). Healthcare activities are generally cooperative ones with a strong distributed component, which is strengthened by the individual representations. Moreover, such cooperation is frequently temporally asynchronous implying changing actors during the patient management by members of a medical team. Medical teams are just a specialization of workers teams defined as a special kind or subset of operator's group; one in which the members have different, though interdependent roles (Salas et al., 1992).

\section{THE EORCA TASK ANALYSIS METHOD}

In this context, our aim was to build a method of observation and representation describing the medical collective activity in various situations as healthcare in an intensive care unit or, in the present case, epidemiological situations. Our focus was first upon specialized physicians' activity, which we hypothesized being a supervised collaboration with others caregivers, members of variable size team. Our goal was to develop an accurate and usable task analysis-oriented tool for observing and modelling medical activities. This method was designed for making explicit the collaborative dimensions of health care as well as human factors involved in decision-making in dynamic, complex and critical safety conditions. The final method had to be robust and reproducible to allow further qualitative and quantitative analysis and also comparative studies. EORCA is based on a three-manual-steps method that allows the representation of the medical team's activities (figure 1):

- Step 1: Observation recording. This step aims at collecting sequences of events by direct observation. It is ruled by definite and mandatory instructions that aim at standardizing observation and recording. These instructions to the observers define how to record observations focusing upon events. An "observed event" is defined as an action performed by an "agentive social object" (Masolo et als, 2003). The agentive social object includes an individual person or a group of persons (a physician, a nurse or others contributors to the patient management). The recorded action may be a deed or a verbal communication occurring between caregivers as explicit decision-making requests or questions. Events are recorded in chronological order with non-systematic timestamps. Semi-directive post-task interviews with physicians about the observed cases complete observations. The result of this step is an eventoriented written description in natural language of patient's care scenario. 
- Step 2: Action codification. This step aims at analysing the scenario for extracting events' components and sequencing. Actions and agents are identified and coded with regard to the domain-oriented ontology, which formalizes and organizes concepts involved in observations. The taxonomy in the ontology is based both upon a yetexisting one, the DOLCE ontology (Gangemi A, 2002) and upon an analysis of data coming from preliminary observations. If needed, the lower ontology classes are completed from observation during this step. The result is a scenario transcription, in which each event component (i.e. action, agent, time, location) is coded and classified in these categories.

- Step 3: Event representation. This last phase consists in describing the coded scenario with an event-oriented formalisation (Chaudet, 2006), which leads to a graphical representation of the observed scenario. We built a representation of the current task world, where each element of the final situational representation is an event occurrence component, identified during the previous step implying the agents and their actions. For this purpose, we have defined graphical generic concepts to build the final representation sheet of the medical management activities. The goal is to have the representation, in a single template, of the main components of medical situation management by the team members.

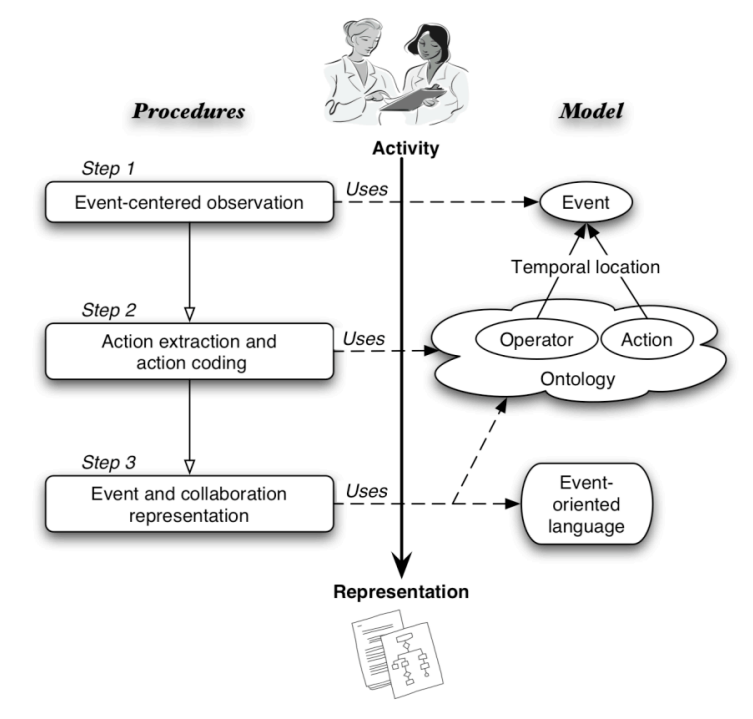

Figure 1: General structure of the EORCA task analysis

\section{Codification of observed actions}

The identification of classes and sub-classes of actions performed by team members would give us information about the medical situation management. Our goal was to identify indicators of collaboration between actors as information transmission, decisions and actions required or transmitted by members of medical team in constrained and risky situations. Studies upon collaborative work teams in medical high-risk situations as trauma patient resuscitation, anaesthesia (Xiao et als, 2003, Manser and Whener, 2002) and in others domains have also identified classes of actions in collaborative team activities (Hutchins et als, 2007), especially ways of communication between team members (Karsenty 2000). Three main classes of actions were created to describe our sets of observations:

- Speech acts between the actors, which refer to oral speeches or written acts (including updating patient record) that have been observed and transcribed in the observation sheet. Several sub-classes were identified such as requests for actions from one actor to another one or explicit decisions of actions on the patient.

- Informative actions, which include actions that require information acquisition and data management to manage the current situation. Specialized tools, medical information systems or databases may support them. We first insert in this class purely medical informative actions as clinical examinations, radiology consultations, monitoring et also general control of situation parameters, observations and verifications.

- Non-informative actions include all actions performed by team members, which brings no specific information during the situation management. Such "acting" actions may be medical deeds and nursing or preparation of the patient's medical equipment in case of patient management, or management of computerized systems and usage of tools (faxes, printer, ...) or others observed activities as arrival and exit of the actors. 


\section{Final representation of the medical team activities}

The formalization of the observed situation will allow us to represent, in a final graphical representation, the main elements of the management by the team members. The result of this step is an event-oriented model of the scenario that may be processed for further analysis. Supplementary to the actions, three others meta-concepts were defined to structure the formal description of the observed situation: object, event and location classes. In addition to main objects of our ontology, we defined graphical generic concepts building order to develop the final representation sheet of the patient management activities as points (the agent performs an action), rectangles (the agent initiates an action), axes (the agents are present or leave the space resolution) and segments (the agent performs an action, alone or collectively). To explicitly represent the classes of actions in the final sheet, we choose the following shapes: ovals for representing non-informative actions, such as therapeutics and medical acts, round corner rectangles for representing informative actions, such as clinical and monitoring investigations; regular rectangles for representing speech acts, i.e. all kinds of information transmissions between actors.

\section{STUDYING A SIMULATED COLLECTIVE OUTBREAK ALERT MANAGEMENT}

\section{Medical context : detecting and evaluating epidemiological risks}

Tasks and activities implied in detection and evaluation of epidemiological risks can be well described by the "classical" NDM descriptors of a complex work situation especially with the following ones: time-pressure, uncertainty and highly dynamic environments, ill-structured problems, shifting, ill-defined, or competing goals, action/feedback loops, high stakes, multiple actors and organizational constraints. In these situations, the process of expert decisionmaking is essentially an understanding of the outbreak situation (identify the accurate outbreak, its main features and plausible evolution with an acceptable confidence level) and to select an accurate course of actions (medical countermeasures with respect to time constraints). Epidemiologists must detect pertinent cues from the situational context fort clarifying their understanding of the current situation (a representation of the probable outbreak) on the basis of their expert knowledge (scenarii, frames of epidemiological situations). Once the outbreak is identified (even if it is not biologically confirmed), the experts build a set of medical responses to prevent the outbreak spread. Such responses can be assimilated to a possible course of action. These medical responses have to be appraised within the field constraints before their effective application. The situation context is continuously changing (new cases may appear with the same symptoms or new ones in other locations). The experts have to deal with these parameters in their final choice of the accurate course of action. Moreover, the epidemiological surveillance for early alert warning is a collective activity, performed by a restricted numbers of specialists in this domain. This basic team of experts may call others physicians if necessary because of their medical specialty. So, this situation implies activities of collaboration between medical actors with different expertises and spatiotemporally distant.

\section{The technical context: The ASTER system}

The targeted system, called ASTER, is a groupware application tailored for early epidemiological early warning within French forces. It allows a near-real time surveillance with a routine delay less them $10 \mathrm{~min}$. This system covers a population of about 7000 servicemen abroad, including deployment in rain forest or in desert areas. It supports physicians engaged in common prescribed goals, which are the surveillance, the early alert and the management of epidemic diseases. ASTER seeks to use existing health data in real time to provide immediate analysis and feedback to those charged with investigation and follow-up of potential outbreaks. Its main functions comprise the geo-referenced collecting of signs and symptoms observed during patients' consultations in deployment locations, the continuous automatic analysis of these data for identifying an epidemiological signal aberration, and the specific information processing devices (statistics, geographical information system...) for supporting the epidemiological situation diagnosis with the aim of providing adequate health intervention proposition within a window of opportunity. This system requires interactions between the mobile and distant users and the proposed services as a form of collaborative and asynchronous activity.

\section{The observation context: the outbreak exercise situation}

We built a wizard of $\mathrm{Oz}$ experiment using an ASTER simulation platform in a scenario involving a natural and an intentional outbreak within French Forces deployed in Tchad. Participants in the experiment were a team of 3 physicians, 2 from the Surveillance Unit and 1 from the Medical Intelligence Unit. Physicians and health commander in the duty area were virtual actors played by the game master. Simulated surveillance data were communicated following a "naturalistic" medical rhythm corresponding to a realistic temporal development of the outbreak in this specific situation, including spatio-temporal, geographical and military constraints. The duration of the resolution was 2 hours. At the end of this exercise, the accurate syndrom was identified and the counter-measures were proposed. Observations were recorded by two ergonomists applying EORCA. The goal of this exercise was to obtain a set of systematized field observations illustrating collaborative features of problem solving and decision making processes between expert physicians. We planned to identify modalities of cooperation between actors as cooperative action, planning and control of the situation. We hoped also to identify some elements of distributed cognition as coordination and allocation of 
tasks between in situ epidemiologists, especially how the participants build a shared problem solving and converge to a collective consensus.

\section{RESULTS}

In this paper, we present the first analysis of the data with the EORCA task analysis method, which concerns the categorization of the observed events during the management of this epidemiological warning. These results are obtained at the end of the step 2 of the method. The complete analysis of this exercise is still running. We aim at illustrating the weight of communication between actors for collaboration in the resolution of medical critical situation, previously described by Moss and Xiao (2004). During the scenario resolution, a total amount of 234 events were recorded during $2 \mathrm{~h} 15 \mathrm{mn}$ of situation management, with a ratio of 1.95 events/minutes. These events concern 244 actions, which have been classified in our 3 main classes (table 1). Actions and events are not overlapped because 10 events are composed by speech acts and informative actions. These main categories are issued from the previous studies in ICU collective patient management (Pellegrin et als, 2007). In the two major classes, speech acts and informative actions, we have reapplied the sub-classes in used ICU studies (see table 2 and table 3 ) to this situation. But, the lowest subclasses of actions are directly issued from the observations of the actual experience. In the same idea, its is not possible to reapply categories of non-informative actions issued from ICU observations to epidemiological situation because medical activities are specific to their own field.

\begin{tabular}{|l|c|}
\hline Main classes & nb of actions \\
\hline Speech Acts & $179(73.6 \%)$ \\
\hline Informative Actions & $56(22.95 \%)$ \\
\hline Non informative Actions & $9(3.69 \%)$ \\
\hline Total & 244 \\
\hline
\end{tabular}

Table 1: Main classes of actions

Speech acts are the most frequent observed actions (73.36\% observed actions). They are followed by informative actions (22.95\% observed actions). Non-informative actions constitute the less observed actions (3,69\% observed actions). A Pearson Chi-squared test for given probabilities were performed with these data, which indicates that this repartition is non aleatory (Chi-squared test for given probabilities: $\mathrm{X}$-squared $=189.5$, $\mathrm{df}=2, \mathrm{p}$-value $<2.2 \mathrm{e}-16$, $\mathrm{R}$ data). In this study, speech acts and informative actions cumulate $96,31 \%$ of the observed actions. These data illustrate the importance of communication in the management of constrained health situations. The detail of speech acts illustrates what kinds of information are transmitted (or needed) between one agent (or a set of agents) to another one to solve conflicted alternatives, to anticipate undesirable consequences and to reduce uncertainty. (table 2).

\begin{tabular}{|l|c|}
\hline Classes of Speech Acts & nb of actions \\
\hline Request for information & $59(30.26 \%)$ \\
\hline Information transmission & $40(20.51 \%)$ \\
\hline Response & $38(19.48 \%)$ \\
\hline Collective Discussion & $22(11.28 \%)$ \\
\hline Request for actions & $18(9.23 \%)$ \\
\hline Decisions (Explicit Decision making) & $18(9.23 \%)$ \\
\hline Total & $195 \%$ \\
\hline
\end{tabular}

- One event in the Speech Acts class could be described by more than one subclass (e.g., a complex event may concern an explicit decision making justifying a request for action).

Table 2: Actions repartition of speech acts in identified classes

In these results, request for information and information transmission concern $50.77 \%$ of the total amount of speech acts, response and collective discussion concerns $30,77 \%$ of these actions and request for actions and explicit decision making $18.46 \%$. They show the range of activities performed by the physicians to deal with the situation (Chi-squared test for given probabilities: $\mathrm{X}$-squared $=40.6, \mathrm{df}=5, \mathrm{p}$-value $=1.13 \mathrm{e}-07$ ). The numerous requests for information 
transmission and responses between medical actors $(70.26 \%$ of the total) illustrate the weight of verbal activity in this situation. During the resolution, utterances are used to manage expectancies, evaluation of plausible epidemiologic situations, pertinence of data, diagnosis, goals to reach and actions to undertake and physicians' efficient responses to the situation, for example, by verbally indicating actions and decisions, or questioning about the affected population and its environment. Management of information by the way of specific undertaken actions complete the speech acts activities performed by the epidemiologists mainly supported by the system ASTER, with an equivalent amount of observed actions as collecting information, monitoring situation, management and validation of obtained information (Chi-squared test for given probabilities, $\mathrm{X}$-squared $=4, \mathrm{df}=3$, $\mathrm{p}$-value $=0.2615$, $\mathrm{R}$ data).

\begin{tabular}{|l|c|}
\hline Classes of Informative Actions & nb of actions \\
\hline Information, data acquisition & $20(35.7 \%)$ \\
\hline Situation monitoring & $14(25 \%)$ \\
\hline Information, data management & $12(21.4 \%)$ \\
\hline Information validation & $10(17.9 \%)$ \\
\hline Total & 56 \\
\hline
\end{tabular}

Table 3: Actions repartition of Informative Actions in identified classes

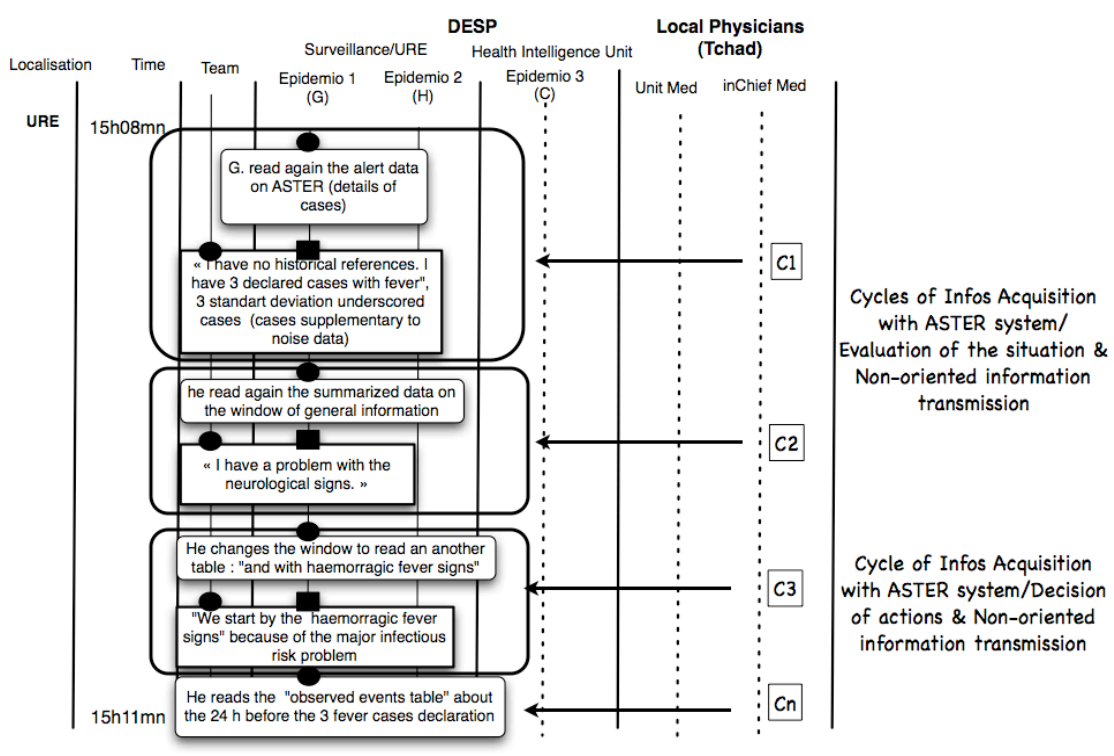

Figure 2: EORCA graphical representation (first evaluation of the situation)

Based upon the cited previous studies (Pellegrin et al., 2007), we have supposed that information transmissions and management between the actors play an important role for actions, adjusting behavior faced to difficulties, and making anticipations and previsions. Even if these two medical situations are different, the both ones (epidemiological alert and ICU patient management) are collective and complex activities, characterized by uncertainty events and complex actions performed by a set of persons in the shortest interval of time. In the present case, physicians build a representation of the epidemiological situation and choose accurate actions thanks to an active information search and transmissions of information between members of the team. The final graphical formalization build with EORCA method shows sequences of actions which illustrates the ways to solve the situation as for example:

- Cycles of information acquisition, helped by the system or by other tools (telephone, internet browsing...) and speech acts (as information transmission) between the 2 epidemiologists during the first $45 \mathrm{mn}$ of resolution (figure 2);

- Packages of actions leading to a situation diagnosis including a collective discussion between actors to evaluation the current state of medical information and the planning of their mutual actions ( 3 epidemiologists, 1 unit physician), a cycle of information acquisition/information transmission including parallel actions and the final confirmation of one pathogen agent (figure 3). 


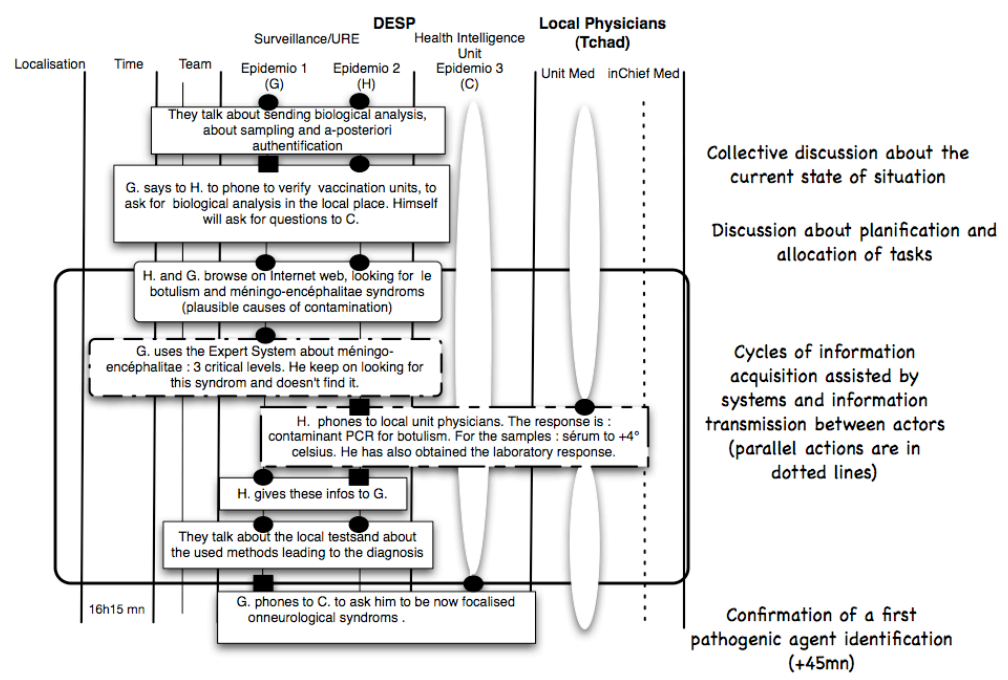

Figure 3: EORCA graphical representation (Building a representation of the current situation)

\section{CONCLUSION}

Thanks to EORCA method, it is possible to have a global view of the main features of these activities as search for contextual data and their collective evaluation to build in a representation of the current situation, which leads to the planning of medical responses and allocation of respective tasks between the main actors of the situation. Theses actions of medical information management performed by the epidemiologists would be linked to the 4 elements of recognition in the NDM model: management of expectancies, search for relevant cues, identification of plausible goals, dentification of typical actions. Because EORCA allows both a categorization of actions and a formalization of events in their temporal and spatial occurrence, we can observed the elements would participate both in the identification of a plausible outbreak as a diagnosis of an "unclear" situation (as the variation 2 of the NDM model) and in the evaluation of the plausible courses of action, health consequences upon the population as time passed with or without medical actions (variation 3 of the NDM model. EORCA is a method using a representational model associated to a set of procedures for building an event-oriented representation of complex medical situations involving several actors. This method helps us to identify, which kind of actions are implied to support the situation management. The results show that this method is able to report the temporal organization of medical situations management and especially the dynamics of communication and collaboration between actors. All these elements are gathered in a descriptive model of the situation that highlights the difficulties of case management linked to outbreak hazard, complexity of the situation, and time constraints. It is then adapted to time-constraint and risky situations where a high level of cooperation and planning is required. We have observed that tasks were jointly performed by a set of caregivers sharing the same objectives and they were based on a mutual and distributed understanding of intentions and actions. The results of this study illustrate the creation of a shared problem representation, implying a strong part of cooperative work in a decision making process. In this context, multiple and sometimes divergent interpretations of problems are possible, and may co-exist among them, This is especially true when actors are confronted with a problem characterized by a high level of complexity and/or lack of accurate data, or are lacking relevant knowledge for fully understand it. At least some problems remain to be solved. The reliability of the categorisation of each action in the main classes and in their respective subclasses has not be validated. We have not yet provided a measure of consistency or reliability with which these codes can be applied between two or more ergonomists participating to such studies. Finally, it will be necessary to find the adequate level of abstraction, actions sequences and events scenarios or macroevents, to interpret the observations with granularity appropriate for a better understanding of the current situation analysis and the building of the course of action (Dojat et al., 1998).

\section{ACKNOWLEDGMENTS}

We thank MCS Jean-Paul Boutin, MC Xavier Deparis from IMTSSA and MC Lionel Bourdon from IMNSSA, which have contributed to the realization of this study. We especially are grateful to the epidemiologists of the Surveillance Unit for their fully participation to this research. 


\section{REFERENCES}

Beuscart-Zephir MC, Pelayo S, Anceaux F, Meaux JJ, Degroisse M, Degoulet P. (2005) Impact of CPOE on doctornurse cooperation for the medication ordering and administration process. Int J Med Inform 2005 Aug;74, 7-8, 62941.

Chaudet H, Pellegrin L, Meynard JB, Texier G, Tournebize O, Queyriaux B, Boutin JP. (2006). Web Services Based Syndromic Surveillance for Early Warning within French Forces. Stud Health Technol Inform, 124, 666-71.

Chaudet, H (2006). Extending the event calculus for tracking epidemic spread. Artificial Intelligence in Medicine, 38(2), 137-156.

Dojat, M., Ramaux, N., Fontaine, D. (1998). Scenario recognition for temporal reasoning in medical domains. Artificial Intelligence in Medicine 14, 139-155.

Fiore S., Salas E., Cuevas H., Bowers C. (2003). Distributed coordination space: toward a theory of distributed team process and performance, Theoretical Issues in Ergonomics Science, 4, n³-4, 340-364.

Gaba DM. (1992). Dynamic decision-making in anesthesiology: Cognitive models and training approaches. In: Evans DA, Patel VL, editors. Advanced Models of Cognition for MedicalTraining and Practice. Heidelberg, Germany: Springer-Verlag, 123-148.

Gangemi A., Guarino N.,. Masolo C, Oltramari A., Schneider L.(2002). Sweetening ontologies with DOLCE. $n$ A. Gómez-Pérez, V.R. Benjamins (eds.) Knowledge Engineering and Knowledge Management. Ontologies and the Semantic Web, 13th International Conference, EKAW 2002, Siguenza, Spain, October 1-4, Springer Verlag. 2002, pp. 166-181

Hutchins, S. Bordetsky, A., Kendall T., Garrity, M. (2007). Empirical Assessment of a Model of Team Collaboration, In Proceedings of NDM 8, Eighth International Conference on Naturalistic Decision Making, 135-142.

Karsenty L. (2000). Cooperative work: the role of explanation in creating a shared problem representation, Le Travail Humain, 63, n4, 289-309.

Klein, G. (1993). A Recognition-Primed Decision (RPD) Model of Rapid Decision Making. In G. A. Klein, J. Orasano, R. Calderwood, \& C.E. Zsambok (Eds.) Decision Making in Action: Models and Methods, Ablex Publishing Corporation, New Jersey, 138-147.

Lipshitz R., Klein G., Orasanu J. , Salas, E. (2001). Focus Article: Taking Stock of Naturalistic Decision Making, Journal of Behavior and Decision Making, 14, 331-352.

Manser T., Wehner T. (2002). Analysing action sequences: Variations in Action Density in the Administration of Anaesthesia, Cognition Technology \& Work, 4, 71-81.

Masolo C., Borgo S., Gangemi A., Guarino N., Oltramari (2003). A. WonderWeb deliverable D18 Ontology Library (Final), IST Project 2001-33052 WonderWeb: Ontology Infrastructure for the Semantic Web, Deliverable.2003, http://wonderweb.semanticweb.org/deliverables/documents/D18.pdf

Moss J, Xiao Y (2004). Improving Operating Room Coordination: Communication Pattern Assessment. Journal of Nursing Administration, 34(2), 93-100.

Nyssen AS, De Keyser V. (1998). Improving Training in Problem Solving Skills: Analysis of Anesthetist's Performance in Simulated Problem Situations, Le travail humain, 61, 387-402.

Patel VL, Kaufman DA, Arocha JF. ( 2002) Emerging Paradigms of Cognition and Medical Decision Making, Journal of Biomedical Informatics, 35, 52-75

Pellegrin L; Bonnardel N; Antonini F; Albanese J; Martin C; Chaudet H. (2007). Event oriented representation for collaborative activities (EORCA). A method for describing medical activities in severely-injured patient management. Methods of information in medicine, 46, 5, 506-15

Reddy, M., Pratt W., Dourish P., Shabot M.M.. (2002). Asking Questions: Information Needs in a Surgical Intensive Care Unit, In Proceedings of American Medical Informatics Association Fall Symposium (AMIA'02). San Antonio TX. Nov 9-13, 657-651.

Salas, E. Dickinson, T. L., Converse, S. A., \& Tannenbaum, S. I. (1992). Toward an understanding of team performance and training. In R. W. Swezey \& E. Salas (Eds.), Teams: Their training and performance, Norwood, NJ: Ablex, 3-29.

Xiao, Y. Hunter, W. Mackenzie, C. Jefferies, N. \& the LOTAS GROUP (1996). Task complexity in Emergency Medical Care and its implications for team coordination. Human Factors, 38, 636-645.

Xiao, Y., Seagull, FJ., Mackenzie, C., Ziegert, J., Klein KJ (2003), Team communication patterns as measures of team processes: exploring the effects of task urgency and shared team experience. Proceedings of the Human Factor and Ergonomics Society 47th Annual Meeting, Denver, Colorado, 1502-1506. 